\title{
Metaplasia intestinal e carcinoma gástrico: correlação com os subtipos histológicos da neoplasia
}

\author{
Intestinal metaplasia and gastric carcinoma: a correlation with the neoplasm histological subtypes
}

\author{
Luiz Gustavo M. Rodrigues ${ }^{1}$ \\ Ana Margarida M.F. Nogueira² \\ Liubiana A. Araújo \\ Paulo Guilherme O. Salles ${ }^{4}$ \\ Sílvia Pimenta de Carvalho \\ Mônica Maria D.A. Cabral ${ }^{6}$
}

\begin{abstract}
unitermos resumo
Estômago

Metaplasia intestinal

Carcinoma gástrico

Lesão pré-cancerosa

Gastrite atrófica

Introdução: O papel da metaplasia intestinal (MI) como lesão pré-cancerosa gástrica não está completamente esclarecido. Objetivos: Analisar a distribuição e o tipo de Ml em relação aos tipos de carcinoma gástrico (CG). Material e Método: Analisaram-se 71 peças de gastrectomia por CG. Amostras da neoplasia e da mucosa a intervalos regulares de toda a peça foram processadas rotineiramente; os cortes histológicos foram corados por HE $\mathrm{e}$ histoquímica para mucinas. A gastrite crônica atrófica (GCA) e a MI foram analisadas quanto a topografia, intensidade e tipo e correlacionadas com o subtipo da neoplasia. Resultados: Diagnosticaram-se 42 (59\%) CGs do tipo intestinal (Cal), 17 (24\%) difusos (CaD) e 12 (17\%) não foram classificáveis. A GCA e a Ml foram mais intensas no Cal do que no $\operatorname{CaD}(p=0,02$; $p=0,004)$. Observaram-se focos de MI dos tipos I, II e III em 21 Cal (50\%), enquanto que 5/17 (29\%) CaD com Ml apresentavam focos de MI de tipos I e II. A MI do tipo III foi diagnosticada em $21 \mathrm{Cal}(50 \%)$ e em seis $\mathrm{CaD}(35 \%)(p=0,04)$; nas áreas peritumorais com Ml, a do tipo III foi detectada em 13/34 (38\%) Cal e 3/10 (30\%) CaD. Houve correlação entre a intensidade da Ml e o tipo da lesão $(p=0,005)$, observando-se MI de tipo III predominantemente quando as lesões eram de intensidade moderada a acentuada. Conclusões: A MI de tipo III pode ser considerada um marcador da intensidade do processo metaplásico. Sua presença em 50\% dos Cal limita seu papel como lesão de risco e reforça a hipótese da existência de diversas vias carcinogênicas para o CG.
\end{abstract}

Recebido em 17/07/01 Aceito para publicação em 14/08/0 


\section{Introdução}

Em 1965, Láuren (18) classificou os tumores gástricos em dois tipos, intestinal e difuso, de acordo com suas características morfológicas, bioquímicas e epidemiológicas. O carcinoma de tipo intestinal (Cal) é o principal tipo de tumor encontrado em populações de alto risco, acomete mais homens que mulheres, com uma incidência de 2:1, e faixa etária em torno dos 60 anos $(4,5,18,19)$. É um adenocarcinoma com diversos graus de diferenciação, cujas células são grandes e claramente definidas, pleomórficas, com núcleos hipercromáticos, apresentando freqüentemente figuras de mitose e invadindo a parede gástri$\mathrm{ca}$, formando predominantemente massas distintas e definidas segundo um padrão de crescimento expansivo. A gastrite crônica atrófica (GCA) e a metaplasia intestinal (MI) estão associadas a este tipo de neoplasia e são consideradas condição e lesão pré-cancerosas, respectivamente $(4,5,7,8,14,15,17,25-28)$. O carcinoma difuso $(\mathrm{CaD})$ ocorre mais precocemente, em torno de 45 a 50 anos, com uma incidência semeIhante em homens e mulheres $(17,18)$. A neoplasia tem padrão celular mais homogêneo, com núcleos monomórficos e regulares, sem formar glândulas, e as células neoplásicas isoladas infiltram a parede difusamente. Mutações do gene da caderina-E foram descritas recentemente em $\mathrm{CaD}$ hereditários e esporádicos $(3,11,12,21)$, mas não se conhece qualquer condição pré-cancerosa ou lesão de risco para este tipo de neoplasia, embora existam indícios de que lesões hiperplásicas podem ser precursoras do $\mathrm{CaD}(1,13)$. Além disso, o $\mathrm{CaD}$ pode surgir em estômagos com GCA e MI (16-18).

A Ml é um processo caracterizado pela presença de epitélio com características morfológicas e bioquímicas do epitélio intestinal (8-10), sendo comum na mucosa gástrica, tanto em condições benignas como malignas $(10,14,15,23,26-28,30)$. Trata-se de uma entidade não-homogênea, apresentando vários padrões de diferenciação e maturação das glândulas metaplásicas $(10,22,24)$, o que permite subclassificála de acordo com características morfológicas, bioquímicas e enzimáticas $(7,10)$. Dentre as várias classificações, duas são particularmente utilizadas: a) a que divide a Ml em tipos completo e incompleto, consoante ocorra "mudança" por completo do epitélio gástrico por epitélio intestinal ou coexistam na lesão características gástricas e intestinais; $b$ ) a que subdivide a Ml em tipos I, II e III, com base em características histoquímicas (Quadro). Na MI do tipo I há células absortivas e secreção de sialomucinas e, muito raramente, de sulfomucinas pelas células caliciformes, correspondendo à MI completa. No tipo II, as células caliciformes secretam sialo e sulfomucinas, as células colunares raramente têm "borda em escova" rudimentar, secretam mucinas neutras e, em menor quantidade, sialomucinas e não há células de Paneth. No tipo III, as células colunares secretam sulfomucinas e maior quantidade de sialomucinas. Nesse tipo são encontradas mais atipias celulares, sendo considerada uma lesão displásica por alguns autores (31). O padrão morfológico da MI de tipos II e III corresponde ao tipo incompleto.

Diversos estudos têm demonstrado uma relação etiopatogênica da Ml com o carcinoma gástrico. A MI do tipo III (incompleta) parece estar mais ligada à morfogênese do CG, constituindo uma lesão de risco para o Cal $(8,9,14,15,27)$. Todavia há controvérsias quanto ao papel da MI de tipo III como lesão précancerosa, particularmente quanto à MI de tipo III com ou sem displasia (31). Para os outros tipos de MI, esta relação não está estabelecida. Por outro lado, em até $15 \%$ dos casos de $\mathrm{CaD}$ pode ser diagnosticada $\mathrm{Ml}$, embora a relação entre o $\mathrm{CaD}$ e os subtipos de $\mathrm{MI}$ não esteja definida. Como no nosso meio não existem estudos sobre a distribuição e o tipo de MI no carcino-

\section{Quadro Colorações histoquímicas para mucinas e respectivos resultados}

\begin{tabular}{lcc} 
Técnica de coloração & Cor & Tipo de mucina \\
PAS/alcian blue & Azul & Sialomucinas \\
$(\mathrm{pH} 2,5)$ & Roxa & Sialomucinas + mucinas neutras \\
& Magenta & Mucinas neutras \\
Diamina férrica/alcian & Marrom & Sulfomucinas \\
blue $(\mathrm{pH} 2,5)$ & Azul & Sialomucinas \\
\hline
\end{tabular}


ma gástrico (CG), realizamos um estudo topográfico, quantitativo e histoquímico da Ml e da GCA em relação aos tipos histológicos de neoplasia.

\section{Material e métodos}

Analisaram-se prospectivamente 71 casos de pacientes gastrectomizados por CG em três hospitais de Belo Horizonte (MG): Hospital das Clínicas da UFMG, Hospital Mário Penna e Hospital Luxemburgo.

As peças cirúrgicas foram colhidas no bloco cirúrgico imediatamente após a cirurgia. No laboratório, foram abertas pela grande curvatura, lavadas e distendidas em telas para fixação com formol a 10\% por 24 horas. Foram retirados fragmentos de $3 \mathrm{~cm} \times 0,5 \mathrm{~cm}$, compreendendo mucosa e submucosa, a intervalos regulares, ao longo da pequena e grande curvaturas $e$ nas paredes anterior e posterior (Figura 1), em toda a superfície da peça cirúrgica. Foram ainda retirados dois a cinco fragmentos representativos da neoplasia. Todo o material foi processado de modo rotineiro; cortes histológicos de $5 \mu \mathrm{m}$ foram corados por hematoxilinaeosina (HE), PAS/alcian blue pH 2,5 e diamina férrica/ alcian blue $\mathrm{pH}$ 2,5 para identificação de mucinas (Quadro) e classificação da $\mathrm{Ml}(7,10)$.

Os carcinomas foram classificados, de acordo com Laurén, em tipos intestinal e difuso (18). Os carcinomas mistos, ou que não preencheram os critérios para classificação em um ou outro tipo, foram considerados não-classificáveis (CaNC). A GCA foi classificada, em cada área, em ausente, discreta, moderada e acen-

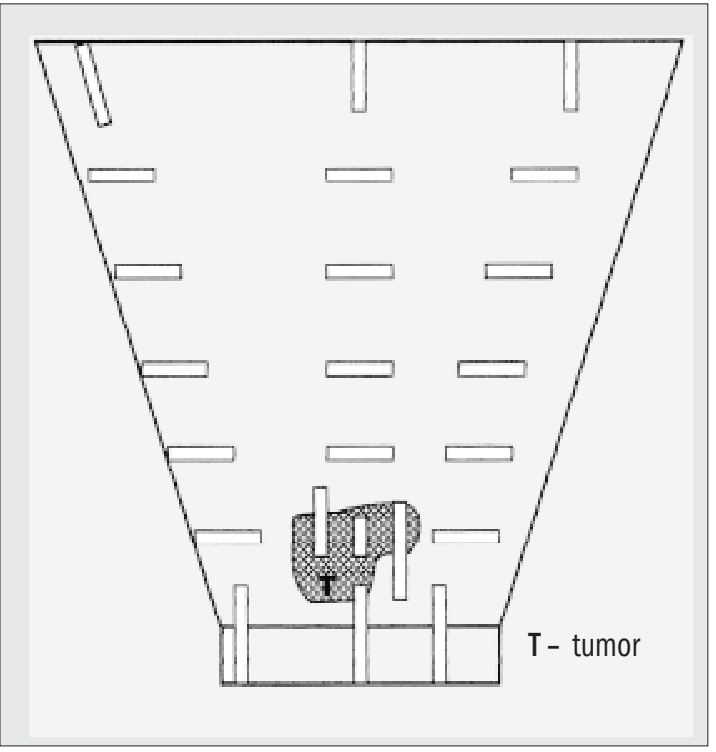

Figura 1 - Mapa da colheita de amostras para estudo histológico em peças de gastrectomia por carcinoma gástrico tuada, de acordo com critérios semelhantes aos adotados na revisão do sistema Sydney (6). Da mesma forma, a MI foi classificada, quanto à intensidade, em: a) ausente; b) discreta - focos pequenos, ocupando até um terço da área examinada; c) moderada - focos maiores, ocupando até dois terços da área examinada; d) acentuada - áreas extensas ocupando mais de dois terços da área examinada (6). Na graduação da intensidade da GCA e da Ml, esta foi classificada em tipos I, II e III $(6,9)$. O tipo, a intensidade, a proporção relativa de cada tipo e a distribuição da Ml foram analisados para cada área. Em cada caso, o resultado foi uma avaliação global média das diversas áreas. Os resultados da GCA e da Ml foram correlacionados com o histotipo da neoplasia.

\section{Resultados}

Foram diagnosticados $42 \mathrm{Cal}$ (59\%), $17 \mathrm{CaD}$ (24\%) e 12 CaNC (17\%). Não se observou diferença, quanto à idade dos pacientes, em relação ao tipo de neoplasia: Cal - $58 \pm 11,6$ anos; $\mathrm{CaD}-57,3 \pm 14,3$ anos; e CaNC $-62,4 \pm 9,9$ anos $(p>0,05)$. O sexo masculino predominou em todos os subgrupos, especialmente no Cal, em que $30(71,4 \%)$ pacientes eram do sexo masculino (2,5 homens: 1 mulher); nos subgrupos difuso e não-classificável, $58 \%$ dos pacientes eram do sexo masculino.

A GCA e a Ml foram observadas independentemente do tipo histológico da neoplasia. Todavia, enquanto a GCA foi igualmente freqüente nos $\mathrm{Cal}$ (39/42; 93\%) e nos CaD (15/17; 88\%) ( $p=0,6)$, a Ml foi significativamente mais freqüente no primeiro grupo $(p=0,006)$. A GCA e a Ml foram mais intensas no Cal do que no $\operatorname{CaD}(p=0,02$ e $p=0,004$, respectivamente) (Figuras 1 e 2).

No que diz respeito ao tipo de Ml, no Cal predominaram casos em que se observaram focos de $\mathrm{Ml}$ dos tipos I, II e III ( $n=21 ; 50 \%)$, enquanto que no $\mathrm{CaD}$ predominaram casos que apresentavam focos de MI de tipos I e II ( $n=5 ; 29 \%)$. A MI do tipo III foi diagnosticada em 21/42 casos (50\%) dos Cal e em $6 / 17$ (35\%) dos $\mathrm{CaD}(p=0,4)$ (Tabela 1). Sete dos 12 casos de CaNC (58\%) apresentavam focos de Ml de tipos I, II e III, e o tipo III foi dignosticado em oito casos (67\%) deste grupo (Tabela 1).

Observou-se Ml em áreas peritumorais de 34/40 (81\%) Cal, 10/17 (59\%) CaD e 10/12 (83\%) CaNC. Nestas áreas metaplásicas observaram-se focos de Ml de 


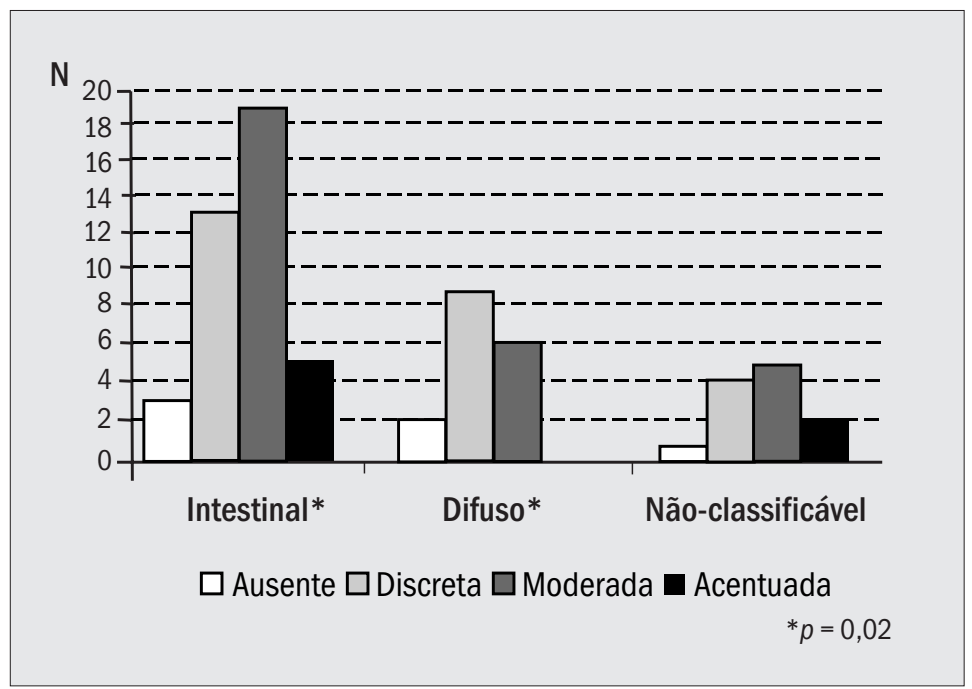

Figura 2 - Intensidade da atrofia em relação ao subtipo histológico da neoplasia

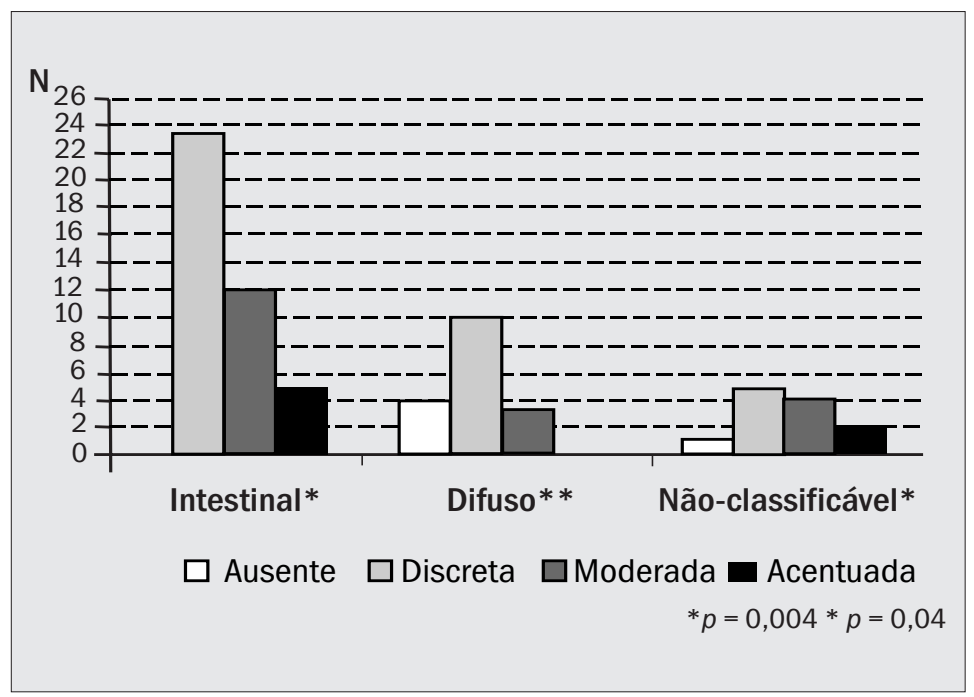

Figura 3 - Intensidade da metaplasia intestinal em relação ao subtipo histológico da neoplasia tipo III, isolados ou associados a outros subtipos, em 13/ 34 (38\%) Cal e 7/10 (70\%) CaNC. Nas áreas peritumorais do CaD observou-se Ml de tipo II em 6/10 casos (60\%) e Ml de tipo III em outros três (30\%) (Tabela 2).

Em relação à proporção relativa de cada tipo de MI (tipo predominante de MI) observado nos focos de Ml de cada caso, o tipo II predominou em 16/42 (38\%) Cal e em 4/12 (33\%) CaNC, e o tipo I predominou em 6/17 (35\%) CaD.

Houve correlação estatisticamente significativa entre a intensidade da Ml e o subtipo histológico da lesão (tipos I, II ou III) (Figura 4). A MI do tipo III foi diagnosticada predominantemente nos casos com MI de graus moderado e acentuado. Além disso, quanto mais extensa a $\mathrm{Ml}$, mais freqüentemente se observaram os diversos subtipos de $\mathrm{Ml}$ em focos intercalados, adquirindo um "padrão de mosaico".

\section{Discussão}

Nos últimos anos tem havido um interesse crescente pelo estudo dos mecanismos envolvidos na carcinogênese gástrica, em parte devido à redescoberta do Helicobacter pylori e à constatação de que a infecção pelo microrganismo é um fator de risco para o $\mathrm{CG}$, tanto Cal como CaD $(4,29)$.

Existem diversos estudos sobre as características do CG e sua associação com lesões e condições précancerosas, especialmente em relação a Cal, GCA, displasia e MI (4, 5, 7-9, 14, 15, 18, 19, 26-28). Todavia há alguns pontos que nunca foram devidamente investigados, bem como aspectos mais controversos,

\section{Subtipos de metaplasia intestinal (MI) em relação ao subtipo histológico}

Tabela 1 da neoplasia

Carcinoma gástrico

\begin{tabular}{|c|c|c|c|c|}
\hline $\begin{array}{l}\text { Tipos } \\
\text { de MI }\end{array}$ & $\begin{array}{c}\text { Intestinal - N (\%) } \\
(n=42)\end{array}$ & $\begin{array}{c}\text { Difuso }-N(\%) \\
\quad(n=17)\end{array}$ & $\begin{array}{c}\text { Não-classif. - N (\%) } \\
(n=12)\end{array}$ & Total \\
\hline Ausente & $0(0)$ & $4(23,5)$ & $1(8,3)$ & 5 \\
\hline Tipo I & $9(21,4)$ & $1(5,9)$ & $1(8,3)$ & 11 \\
\hline Tipo II & $4(9,5)$ & $1(5,9)$ & $1(8,3)$ & 6 \\
\hline Tipos I + II & $8(19)$ & $5(29,4)$ & $1(8,3)$ & 14 \\
\hline Tipos I + III & $0(0)$ & $2(11,8)$ & $0(0)$ & 2 \\
\hline Tipos II + III & $0(0)$ & $1(5,9)$ & $1(8,3)$ & 2 \\
\hline Tipos I + II + III & $21(50)$ & $3(17,6)$ & $7(58,3)$ & 31 \\
\hline
\end{tabular}

Não-classif. = não-classificável 
Subtipos de metaplasia intestinal (MI) na mucosa peritumoral em relação Tabela 2 ao subtipo histológico da neoplasia

\begin{tabular}{lcccc} 
& \multicolumn{4}{c}{ Carcinoma gástrico } \\
Tipos & $\begin{array}{l}\text { Intestinal }-\mathrm{N}(\%) \\
\text { de MI }\end{array}$ & $\begin{array}{c}\text { Difuso - N }(\%) \\
(\mathrm{n}=42)\end{array}$ & $\begin{array}{c}\text { Não-classif. }-\mathrm{N}(\%) \\
(\mathrm{n}=17)\end{array}$ & Total \\
Ausente & $8(19)$ & $7(41,2)$ & $2(16,7)$ & 16 \\
Tipo I & $5(11,9)$ & $1(5,9)$ & $1(8,3)$ & 7 \\
Tipo II & $10(23,8)$ & $3(17,6)$ & $1(8,3)$ & 14 \\
Tipos I + II & $6(14,3)$ & $3(17,6)$ & $1(8,3)$ & 10 \\
Tipos I + III & $0(0)$ & $2(11,8)$ & $1(8,3)$ & 3 \\
Tipos II + III & $9(21,4)$ & $1(5,9)$ & $6(50)$ & 16 \\
Tipos I + II + III & $4(9,5)$ & $0(0)$ & $0(0)$ & 4 \\
\hline
\end{tabular}

Não-classif. = não-classificável

principalmente em relação ao papel da Ml como lesão pré-cancerosa (31). Tendo em vista estas questões e considerando que o CG é muito comum no nosso meio, e que não há estudos que abordem detalhadamente a relação da Ml com o CG, decidimos fazer um estudo topográfico, quantitativo e qualitativo da MI no CG.

Nossos dados são semelhantes ao relatado na literatura quanto ao tipo de CG predominante (Cal), freqüência em relação ao sexo e média de idade dos pacientes. O Cal é o mais freqüente na nossa população, o que está de acordo com o observado em populações de alta e média prevalências de CG (5). Embora os casos estudados tenham sido selecionados, a proporção relativa dos diversos tipos corresponde ao observado geralmente no nosso meio. O Cal é mais freqüente (59\% no presente estudo), seguindo-se o CaD (24\%) e o CaNC (17\%), este último correspondendo, na maioria da vezes, a tumores mistos.

No estudo que serviu de base à proposta de uma nova classificação para o CG, Laurén relatou que a GCA e a Ml eram muito mais freqüentes no $\mathrm{Cal}$ do que no $\mathrm{CaD}$, porém observou $\mathrm{Ml}$ em $15 \%$ de casos deste último tipo de neoplasia (18). Estudos posteriores mostraram uma clara relação entre MI com secreção de sulfomucinas (tipo incompleto ou tipo III) e Cal, e demonstraram que a MI de tipo III está relacionada com o risco aumentado para desenvolver CG $(4,7-9,14,15,22,25-28)$. Permanece por esclarecer se a MI de tipo III é uma lesão pré-cancerosa no sentido de o câncer evoluir a partir de glândulas metaplásicas, com ou sem displasia, ou se é uma condição que favorece o surgimento de mutações na mucosa gástrica e posterior malignização.

Observamos GCA e Ml em todos os subgrupos de CG, mas a GCA e a Ml foram significativamente mais freqüentes e intensas no $\mathrm{Cal}$ do que no $\mathrm{CaD}$, o que está de acordo com o relatado habitualmente $(4,16$, $18,29)$. O Cal surge, na grande maioria das vezes, em estômagos com GCA e com MI $(16,18)$, que na maior parte dos nossos casos era de grau moderado. Todavia, num pequeno grupo de casos de Cal, a GCA e a Ml eram de grau discreto ou ausentes. Em outro estudo, em que analisamos o fenótipo, gástrico ou intestinal, observamos que há um grupo de Cal que mantém o fenótipo gástrico, à semelhança do grupo de $\mathrm{CaD}$, e um grupo de Cal que perde o fenótipo gástri-

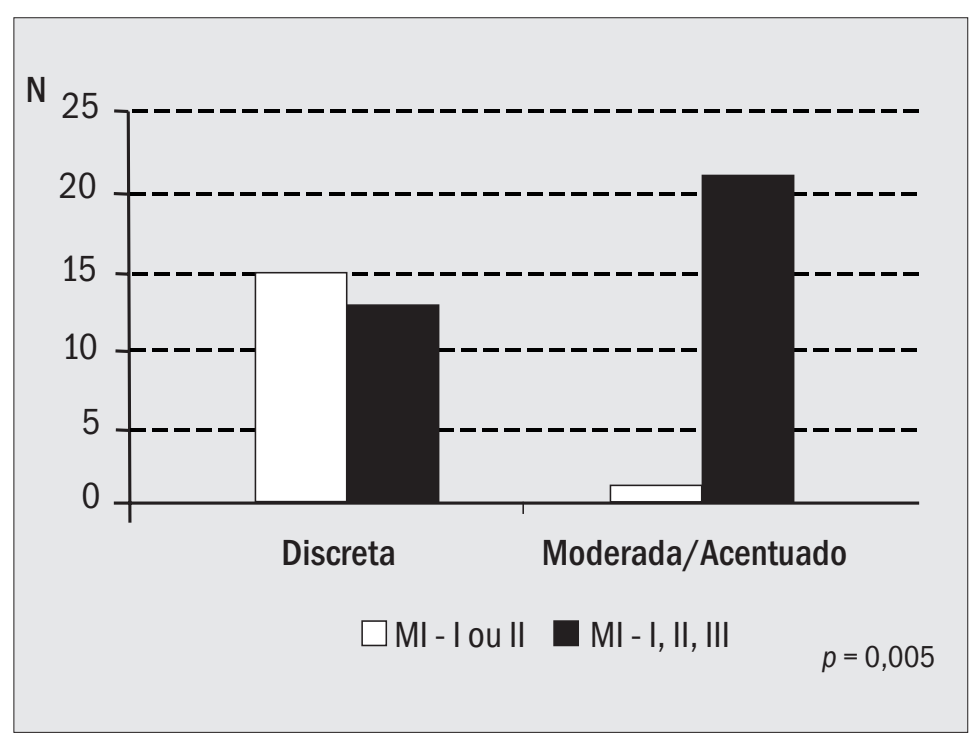

Figura 4 - Relação entre os subtipos e a intensidade de metaplasia intestinal (MI) 
co, adquirindo ou não expressão fenotípica intestinal (20). Estes dados sugerem que nem todos os Cal seguem a mesma via carcinogênica (20), o que de certa forma nossos resultados atuais reforçam, uma vez que a GCA e a MI não eram significativas em uma parte dos Cal, e um pequeno número de $\mathrm{CaD}$ associava-se a $\mathrm{Ml}$ de intensidade discreta e moderada. A idéia de que o CG apresenta fenótipo intestinal tem servido de base para correlacionar o $\mathrm{Cal}$ com a $\mathrm{Ml}$, o que pode não ser correto $(3,20)$. No futuro será interessante estudar a expressão fenotípica destes tumores em relação às alterações da mucosa gástrica, para melhor compreensão das vias patogênicas do CG, neoplasia caracteristicamente muito heterogênea (3).

No tocante aos tipos de Ml verificamos que a de tipos II e III (incompleta) estava presente na mucosa gástrica da maior parte dos casos, sem relação estatisticamente significativa com o subtipo histológico da neoplasia. No entanto a MI de tipo III era mais freqüente no $\mathrm{Cal}$ do que no $\mathrm{CaD}$, embora não significativamente, e na maior parte dos casos as lesões apresentavam-se em focos múltiplos, sendo vistos lado a lado os diversos tipos, como um padrão misto ou em "mosaico". Estes aspectos já tinham sido relatados por outros autores (29), embora não em estudos sistematizados de toda a mucosa como o nosso. Decidimos, portanto, avaliar a proporção relativa dos diversos tipos de Ml verificando o tipo que predominava (em extensão) para cada caso. Observamos que nos Cal os focos de Ml eram predominantemente de tipo II, enquanto que no CaD observava-se, em maior extensão, MI de tipo I. Estas diferenças podem estar relacionadas com a intensidade do processo, como discutiremos adiante, que é diferente nos dois grupos, mas podem ter um papel importante no processo da carcinogênese para os diferentes tipos de neoplasias.

Para avaliar a relação mais estreita da MI de tipo III com os diversos tipos de CG, analisamos o tipo de Ml na mucosa gástrica peritumoral. À semelhança do que tinha sido observado em toda a mucosa, a MI de tipos II e III em torno do tumor não se correlacionou com o tipo histológico do tumor. Ao considerarmos apenas o tipo III, verificamos que era o tipo predominante nos $\mathrm{CaNC}$ e que era observado mais freqüentemente no $\mathrm{Cal}$ do que no $\mathrm{CaD}$, mas a diferença não era significativa. Estes dados sugerem que, contrariamente ao que tem sido relatado, apenas numa porcentagem de casos de Cal parece haver relação direta entre a MI de tipo III e o processo de transformação maligna. Deve ser considerado que a maior parte dos casos que analisamos era de neoplasia avançada e que, portanto, cresceu sem deixar vestígios da mucosa inicialmente relacionada com o processo de malignização. Este é o ponto de vista da maior parte dos autores que estudaram o assunto, mas só a análise destes aspectos em tumores precoces pode esclarecer a questão. Por outro lado, é interessante observar que a relação entre Ml de tipo III e CG de padrão misto foi altamente significativa, e este aspecto deve ser avaliado em maior profundidade no futuro. Finalmente, observamos $\mathrm{Ml}$ de tipo III em torno de $\mathrm{CaD}$, mas o pequeno número de casos não permite estabelecer um elo entre as lesões.

Avaliando em conjunto a relação da MI de tipo III em toda a mucosa gástrica e em torno do tumor, nossos dados corroboram os de outros pesquisadores (4, $8,14,27)$ apenas em subgrupos de Cal e de GC mistos com os quais a lesão está fortemente relacionada. Considerando que este tipo de MI não foi identificado na periferia do tumor em 29 casos (69\%) de Cal, é possível que a $\mathrm{Ml}$ de tipo III seja preferencialmente um marcador de risco, e não verdadeiramente uma lesão pré-cancerosa, como defendem alguns autores $(4,31)$. São necessários estudos de patologia molecular para pesquisa de mutações nesta lesão para melhor avaliação. Por outro lado, sua alta prevalência em torno de CaNC sugere um papel direto no processo, que deverá ser mais bem investigado. A presença de Ml de tipo III num subgrupo de CaD merece ser estudada, com maior número de casos. É possível que esta situação corresponda aos $\mathrm{CaD}$ que se desenvolveriam a partir de mutações em fases precoces de desenvolvimento de Cal (29).

Finalmente, observamos uma relação significativa entre a intensidade da Ml e a presença de MI de tipo III, lesão que não detectamos isoladamente e menos freqüente nos casos com MI discreta. Além disso, quanto mais intenso o processo metaplásico, mais freqüentemente observamos diversos tipos de Ml, o que já foi relatado por alguns autores (29). Observamos focos de MI de tipos I, II e III em áreas adjacentes, que denominamos de padrão "em mosaico". A Ml de tipo II foi o tipo observado na maior extensão dos focos metaplásicos quando a lesão era preferencialmente de grau moderado, e nos casos com Ml discreta observou-se predominantemente o tipo I. Estes dados pa- 
recem sugerir uma progressão dos tipos de $\mathrm{Ml}$ em relação à intensidade da Ml: tipo I > tipo II > tipo III.

Em conclusão, nossos dados mostraram que a MI está fortemente relacionada com Cal e CaNC (maioria de tipo misto) e é muito freqüente em um subgrupo de $\mathrm{CaD}$. Por outro lado, a MI de tipo III pode ser considerada um marcador de intensidade do processo metaplásico. Sua presença em $50 \%$ dos Cal limita seu papel como lesão de risco e reforça a hipótese da existência de diversas vias carcinogênicas para o CG.

\section{Agradecimentos}

Os autores agradecem aos cirurgiões do Hospital das Clínicas da UFMG, Hospital Mário Penna e Hospital Luxemburgo e ao dr. José de Souza Andrade (patologista), por cederem as peças cirúrgicas para o estudo; ao prof. Alfredo J. A. Barbosa, APM, FM/UFMG, em cujo laboratório foi realizada parte do processamento histológico de alguns casos; e a Renata S. Dias, bolsista de apoio técnico (CNPq).

\section{Referências}

1. Becker, K.F. et al. E-cadherin gene mutations provide clues to diffuse type gastric carcinomas. Cancer Res., 54 : 3845-52, 1994.

2. Carneiro, F. et al. Carcinoma arising in gastric hyperplastic polyps. Gastrointest. Endosc., 41: 178, 1995.

3. Carneiro, F. et al. New elements for an updated classification of the carcinomas of the stomach. Pathol. Res. Pract., 191: 571-84, 1995.

4. Correa, P. Human gastric carcinogenesis: a multistep and multifactorial process - First American Cancer Society Award Lecture on Cancer Epidemiology and Prevention. Cancer Res., 52: 6735-40, 1992.

5. Correa, P. The epidemiology of gastric cancer. World J. Surg., 15: 228-34, 1991.

6. Dixon M.F. et al. Classification and grading of gastritis: the updated Sydney system. Am. J. Surg. Patthol., 20(10): 1161-81, 1996.

7. Filipe, M.I. \& Jass, J.R. Intestinal metaplasia and cancer risk. In: Filipe, M.I. \& Jass, J.R. (eds.) Gastric Carcinoma. Edinburgh: Churchill Livingstone, 1986, p. 87.

8. Filipe, M.I. et al. Intestinal metaplasia types and the risk of gastric cancer: a cohort study in Slovenia. Int. J. Cancer, 57: 324-9, 1994.

9. Filipe, M.I. et al. Incomplete sulphomucin-secreting intestinal metaplasia for gastric cancer. Preliminary data from a prospective study from three centers. Gut., 26: 1319-26, 1985.

10. Filipe, M.I. \& Ramachandras, S. The histochemistry of intestinal mucin: changes in disease. In: Whitehead, R. (ed.) Gastrointestinal and esophageal pathology. London: Churchill Livingstone, 1995, p.73.

11. Gayther, S.A. et al. Identification of germline E-cadherin mutations in gastric cancer families of European origin. Cancer Res., 58: 4086-9, 1998.

12. Guilford, P. et al. E-cadherin germline mutations in familial gastric cancer. Nature, 392: 402-5, 1998.
13. Hattori, T. Morphological range of hyperplastic polyps and carcinomas arising in hyperplastic polyps of the stomach. J. Clin. Pathol., 38: 622-30, 1985.

14. lida, F. \& Kusama, J. Gastric carcinoma and intestinal metaplasia: significance of type of intestinal metaplasia upon development of gastric carcinoma. Cancer, 50: 2854-8, 1982.

15. Jass, J.R. Role of intestinal metaplasia in the histogenesis of gastric carcinoma. J. Clin. Pathol., 33: 801-10, 1980.

16. Komoto, K. et al. Helicobacter pylori infection and gastric neoplasia: correlations with histological gastritis and tumor histology. Am. J. Gastroenterol., 93: 1271-6, 1998

17. Koshida, Y. et al. Association of Helicobacter pyloridependent gastritis with gastric carcinomas in young Japanese patients: histopathological comparison of diffuse and intestinal type cancer cases. Histopa thology, 37: 124-30, 2000.

18. Laurén, P. The two histological main type of gastric carcinoma: diffuse and so-called intestinal type carcinoma: an attempt at a histoclinical classification. Acta Path. et Microbiol. Scand., 64: 31-49, 1965.

19. Laurén, P. \& Nevalainem, T.J. Epidemiology of intestinal and diffuse types of gastric carcinoma: a time-trend study in Finland with comparison between studies from high and low-risk areas. Cancer, 71: 2926-33, 1993.

20. Machado, J.C. et al. Gastric carcinoma exhibits distinct types of cell differentiation: an immunohistochemical study of trefoil peptides (TFF1 and TFF2) and mucins (MUC1, MUC2, MUC5AC, and MUC6). J. Pathol., 190: 437-43, 2000.

21. Machado, J.C. et al. E-cadherin gene mutations provide a genetic basis for the phenotypic divergence of mixed gastric carcinomas. Lab. Invest., 79: 459-65, 1999. 
22. Metsukuma, A. et al. Sulphomucin-secreting intestinal metaplasia in the gastric mucosa. Cancer, 66: 689 94,1990.

23. Morson, B.C. Intestinal metaplasia of the gastric mucosa. Br. J. Cancer, 9: 365-76, 1955.

24. Reis, C.A. et al. Intestinal metaplasia of human stomach displays distinct patterns of mucin (MUC1, MUC2, MUC5AC, and MUC6) expression. Cancer Res., 59: 1.003-7, 1999.

25. Silva, S. \& Filipe, M.I. Intestinal metaplasia and its variants in the gastric mucosa of Portuguese subjects: a comparative analysis of biopsy and gastrectomy material. Hum. Pathol., 17: 988-95, 1986.

26. Sipponen, P. Intestinal metaplasia and gastric carcinoma. Ann. Clin. Res., 13:139-43, 1981.

27. Sipponen, P. et al. Intestinal metaplasia with colonictype sulphomucinas in the gastric mucosa; its association with gastric carcinoma Acta Path. et Microbiol. Scand. (Sect. A), 88: 217-24, 1980.

28. Sipponen, P. et al. Atrophic chronic gastritis and intestinal metaplasia in gastic carcinoma: comparison with a representative population sample. Câncer, 52: 1.062-8,1983.

29. Solcia, E. et al. Intestinal and diffuse gastric cancer arise in a different background of Helicobater pylori gastritis throught different gene involvement Am. ). Surg. Pathol., 20: S8-S22, 1996.

30. Stemmermann, G.N. Intestinal metaplasia of the stomach: a status report. Cancer, 74: 55664,1990 .

31. Tosi, P. et al. Gastric intestinal metaplasia type III cases are classified as low-grade dysplasia on the basis of morphometry. J. Pathol., 169: 73-8,1993.
Endereço para correspondência Ana Margarida M.F. Nogueira Depto. de Anatomia Patológica e Medicina Legal (Laboratório de Patologia Cástrica), Faculdade de Medicina - UFMG Av. Alfredo Balena 190 50 andar/Sala 5012 CEP 30130-100 - Belo Horizonte-MG e-mail: anog@medicina.ufmg.br 